\title{
A highly integrated Horizontal coordinate-based tool for architecture
}

\author{
Chi-Li Cheng ${ }^{1}$, June-Hao Hou ${ }^{2}$ \\ ${ }^{1,2}$ National Chiao Tung University, Hsinchu, Taiwan \\ 1micky@arch.nctu.edu.tw2jhou@arch.nctu.edu.tw
}

In this research, we attempt to develop a tool which integrates certain common geographic information from OpenStreetMap and OpenTopography into Grasshopper. We name it as OSMKIT temporarily. Besides, in order to make the integration in the design process easier, this tool includes the bilateral conversion function of coordinate in Rhinoceros $3 D$ and the coordinate of the World Geodetic System. These characteristics bring about several possibilities for further usage. This paper contains explanations of functions and examples. For instance, it can be employed for data visualization on a map when these data contain coordinate information. Additionally, since this tool is simple and intuitive to convert points into GPS coordinates, it can make users plan drone for photogrammetry and deal with other related tasks on the rhinoceros $3 D$ interface, helping them to gain most current urban models. Moreover, architects or designers can be not only users but also contributors for open source map system such as OpenStreetMap; the process of sharing the mode which user measure is demonstrated in this paper. To sum up, this coordinate system based tool is designed to be multifunctional and suitable for interdisciplinary usages in grasshopper.

Keywords: open-source maps, data visualization, geographic information system, urban research, parametric design, interdisciplinary

\section{INTRODUCTION}

Since the data-driven Design process is played a crucial role in the contemporary architecture field. Overlaying various data on the map is a handy way for research or site(Jung Hoon et al. 2013). Nevertheless, Architects usually struggle with integrating abundant and various information and software, seeking the solution everywhere. Certain researchers in architecture engaged in promoting WebGIS(Antje et al. 2018) and relative planning tool(Daniel et al. 2018) for decision making cross scales and disciplines in secondary schools and more and more architects are interested in it; besides, the framework integrating and visualizing GIS-related big data has been proposed(Chen et al. 2014). Since these data available, it provides a whole new perspective of view by visualizing them(Dutt et al. 2016).On the other hand, as the progress of the measurement technique, architects or designers begin to pay attention to the potential of using these techniques in the design process(Danil et al. 2018). By now, either scholars nor architects agree with the importance of the role of data. For in- 
stance, the case of the research about utilizing open data for analysis of livability of urban space manifests the power of it(Eleanna et al. 2017). Additionally, photogrammetry and drone techniques become more and more common and more and more researchers improve relative technology; it seems suitable and practical for measurement for either large scale(Faine et al. 2015) or focusing on one subject(Demetrios et al. 2015). The appearance of various digital tools was a good start to enhance architects. Even though it seems powerful that architects have begun using parametric design tool, it brings about even more challenge that architects have required even more complicated analyses during the design process and the presentation because clients view current architects are capable and responsible for analyzing with computation. The difficulty and complexity of integrating various data can be seen in the relative research which involves BIM, Big Data, CityGML, etc(Jungrim et al. 2015). The reason why the integration of complex information in the design process is always a big issue can arguably be speculated that the situation results from the fact that the majority of design software is designed without the architect's perspective; somehow they are barking up the wrong tree. Those functions and interfaces are either too complex or confusing for architects who are in the design process. As a result, these vain requires that architects can not conquer results in chaos in the architect field. On the other hand, in the view of the fact that architects have started to be used to utilizing the parametric design tool, a parametric tool that manages certain most common and open geographic information simultaneously might work for facilitating architectural design processes.

\section{RELATED WORK}

Certain tools are already existed for gaining model from open-source maps or converting location system. However, they are not easy to integrate them together. First of all, the Elk, which developed by Timothy Logan, is a tool to generate the map and the topographical surface from OpenStreetMap. It is a powerful tool that contains the completed selection function for picking points of particular features, but a fly in the ointment was that Elk hasn't the function of dealing with the conversion of points and Geographic coordinates. On the other hand, the tool MEERKAT GIS, which is developed by Nadlowe, contains the conversion function; However, it is SHP format based tool. The majority of this kind of data is not open and not accessible. Besides, MEERKAT GIS is no more updated, being incompatible with the newest version rhinoceros 3D. Even when they work, it is inconvenient and sophisticated to overlay the models generated from these two tools. It makes spatial data visualization in the urban model problematic. Additionally, @it is a tool that is capable of dealing with OSM format and SHP format. Nevertheless, some tools of it are no more functional; besides, it is quite complex to use them. Therefore, It is time to figure out the new solution for relative tasks or research. Additionally, the solution to reducing the threshold of contributing models is also taken into account.

\section{METHOD}

The goal of developing this tool is facilitating the integration of various information; the fundamental capability is to meet the most general need. Hence, the development started from importing the street map and terrain as base data. Besides, in order to make it easy to adapt. This tool is developed for the most common platform: Grasshopper and all of the functions are collected in one single component as versatile as a swiss army knife. As a result, users just need to focus on only one component and various tasks will be solved by it. The first step to use it is inputting file from OpenStreetMap or Opentopography. Then, the map or terrain will appear in the interface; subsequently, this tool will enable the function of the coordinate converter according to the map. From then on, users are able to handle the conversion between Points in Rhinoceros 3D and coordinate system from other sources freely; besides, it is a crucial part of this tool which makes it multifunctional.

For instance, the further function of this tool is 
Figure 1

Applications of this tool.

Figure 2

This component is with pop up function.

Figure 3 Displaying the map of OpenStreetMap.

Figure 4 The function to select particular models by key.

Figure 5

The example of generating city model.
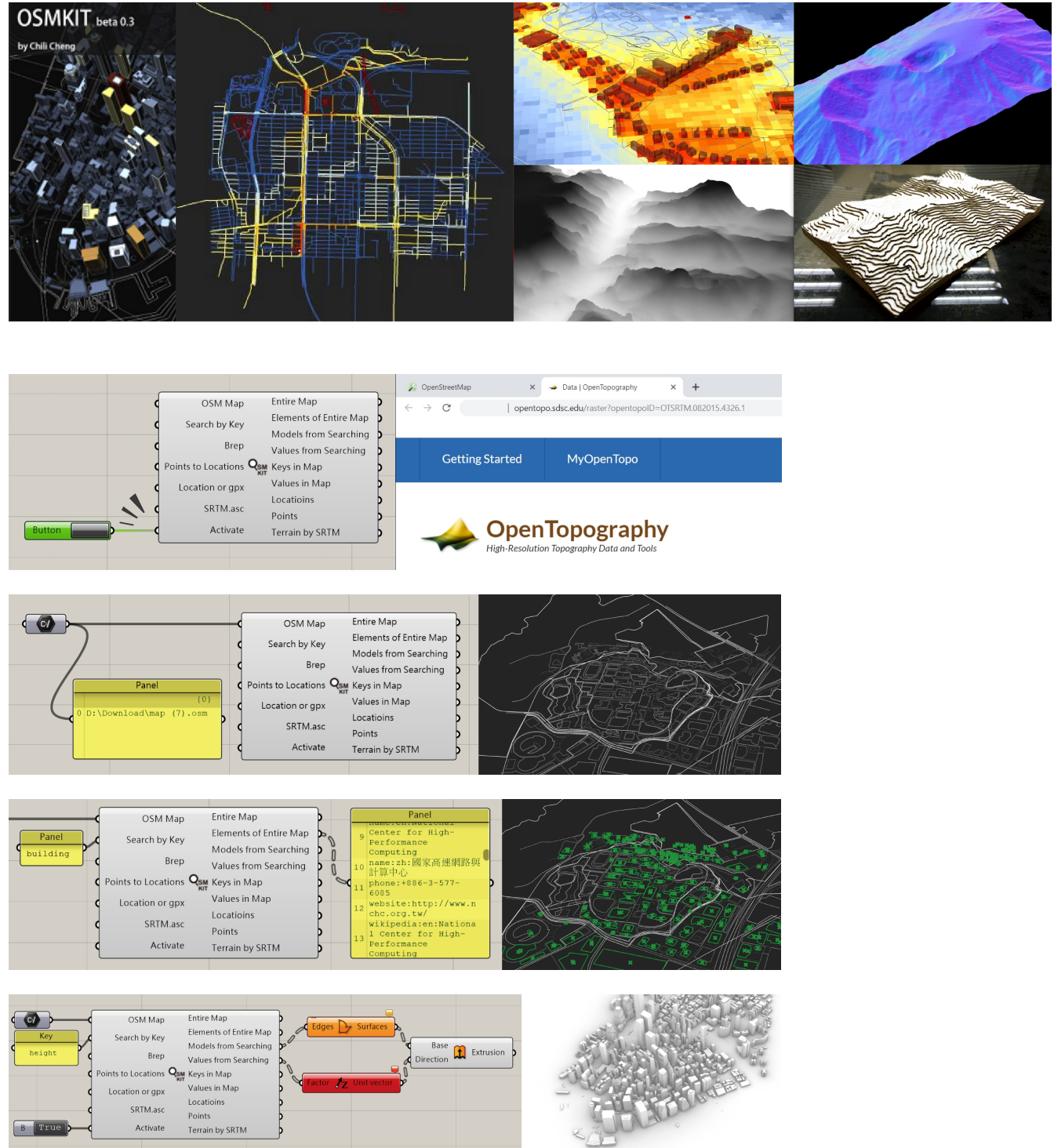
to convert polyline of building into the format that is used for uploading onto OpenStreetMap. The usage which contains converting series points to Drone Path Planning in Grasshopper interface. The utilization of tagging information into the map for spatial data visualization and spatial analysis (Figure 1). In sum, there are various handy usages could be based on the conversion mechanism. The functions and workflow of this tool will be mentioned in the following.

\section{FUNCTIONS}

POP UP. The pop-up function is employed in this tool. It will be triggered when the user doesn't input map or terrain but just activate it. Then, the two websites of OpenStreetMap and Opentopography will pop up immediately and simultaneously(Figure 2), implying that users can gain data from them.

OpenStreetMap. There are three inputs serve the application of using OpenStreetMap, including OSM Map, Search by Key, and Brep. Besides, six outputs for it, such as Entire Map, Elements of Entire Map, Models from Searching, Values from Searching, Key in Map, and Values in Map. Once the map which is downloaded from OpenStreetMap as OSM format is inputted, the polylines of the map will appear instantaneously(Figure 3).

However, the polylines which just appear are not classified. If the user wants to select certain polylines that belong to a particular key, the output Elements of Entire Map will output all of the keys included in the map. Thus, the user can input the key needed into the input Search by Key; then, the polylines of the key will be outputted from Models from Searching(Figure 4). The values correspond to keys will be available as well.

The following is the demonstration of generating the model of Manhattan(Figure 5). In order to make the data flow as simple as possible, it keeps the data structure. Nonetheless, It might result in warning messages because certain data are filtered out by the Search by Key. On the other hand, these data after filtering are correspondent with each other; there- fore, the user doesn't need to worry about it but link these components intuitively.

Coordinate Conversion. Because the data from OpenStreetMap is globally accessible, it is appropriate to be used as the background map. The functions of the location are based on the map from OpenStreetMap. These points in Grasshopper through this tool will be related to Geographic coordinate system. Users are able to derive Geographic coordinate from the map. In this case, it demonstrates the visualization with tagging texts of longitudes and latitudes at points(Figure 6). These functions have a variety of applications, such as data visualization, spatial measurement, and even planning path of a drone.

Furthermore, this function serves to convert Geographic coordinates to points in Grasshopper. It can be utilized for visualization of sorts of data with Geographic coordinates. For example, the data of water quality form Data.Taipei can be automatically tagged on the map in Grasshopper(Figure 7).

Opentopography. This tool contains the function of converting the ASC file from Opentopography into mesh model in grasshopper(Figure 8). The mesh model is outputted from Terrain by SRTM.

Visualizing the GPX format and geographic information. GPX is a common format for tracking path. There are various applications that users can utilize them on their mobile devices. The user can link up the file path of the GPX file to the input (Location or gpx). Besides, this tool allows users to input the file path of GPX format and the list of Geographic coordinate in Grasshopper. It contains the mechanism to recognize what the data is. To do so, the user can use this tool to visualize and convert them into points with visualization of elevation by color in Grasshopper(Figure 9).

Contributing building model onto OpenStreetMap. In order to facilitate the usage of contributing building models, this tool contains the function that converts the Brep model of building into the OSM format for JOSM. In addition, this tool will check whether the models from rhinoceros 3D have inter- 
Figure 6

Visualization of the locations by texts.

Figure 7

The visualization of water quality in Taipei city.

Figure 8

Generating the terrain.

Figure 9

Visualization of GPX data.

Figure 10

The models from Rhino to JOSM.
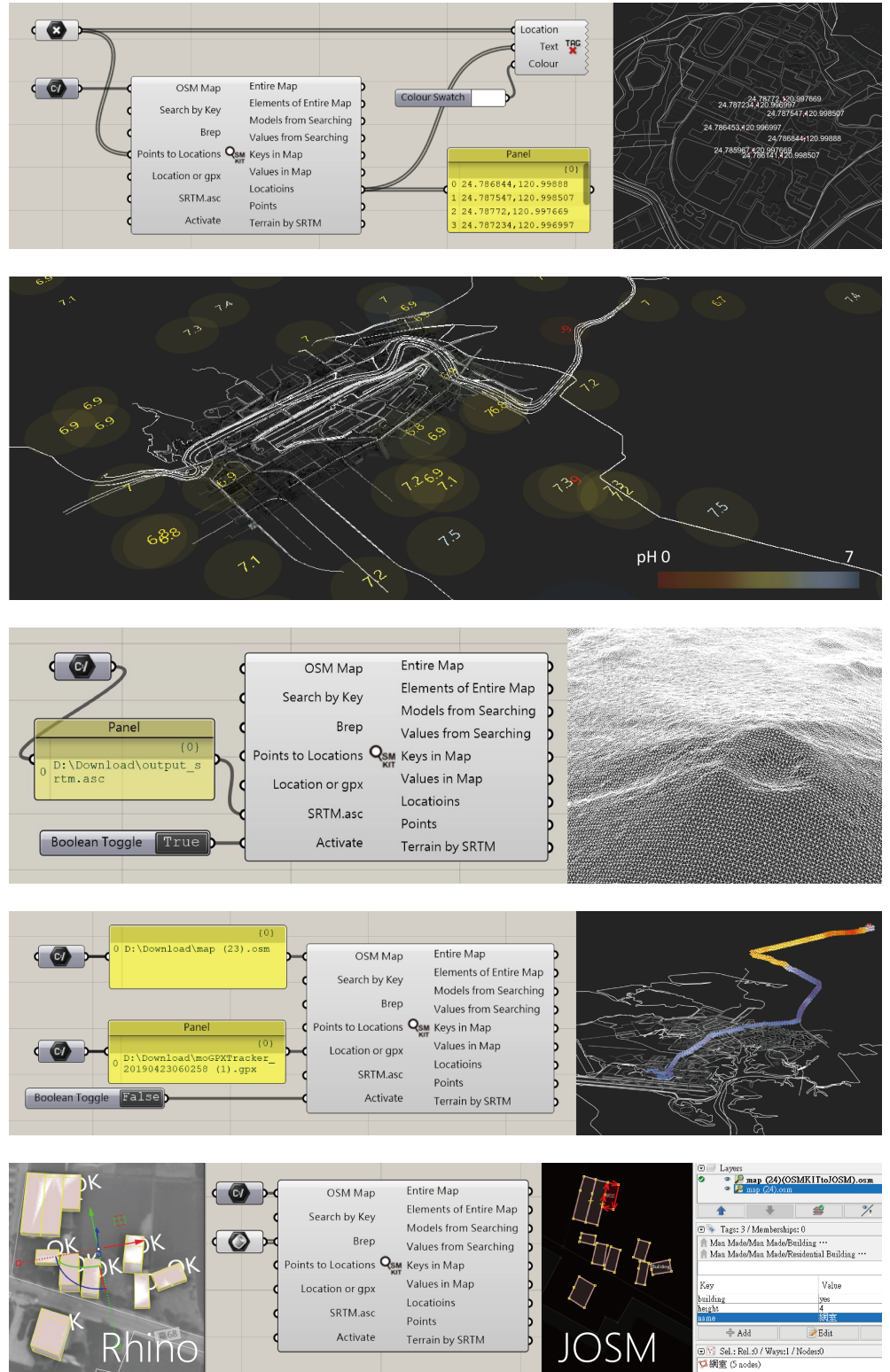

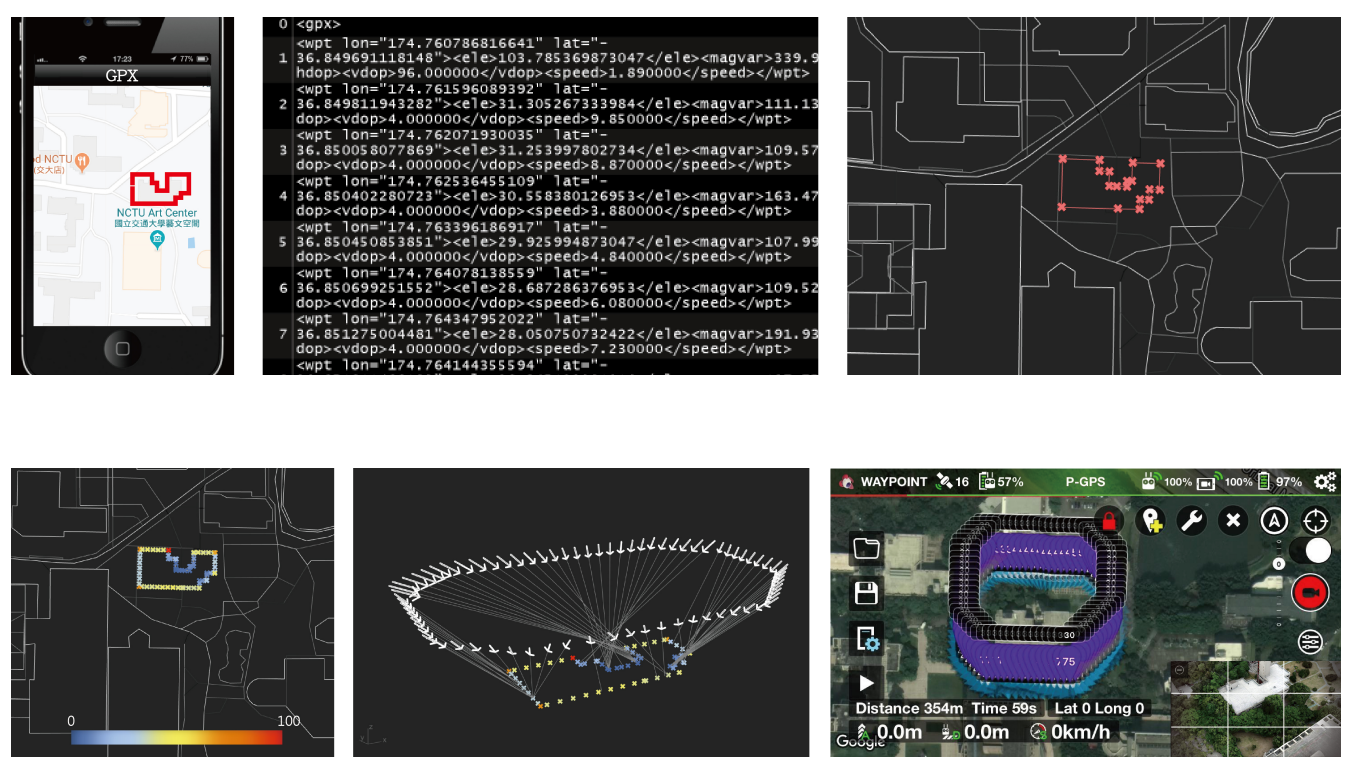

ference with the background map for avoiding overlap. In JOSM, users are able to add more information and then upload them onto OpenStreetMap(Figure 10).

DRONE PATH PLANNING. After elaborating these functions, the demonstration about integrating various data for photogrammetry is described here. In research or a design process, the acquisition of models plays a vital role. Besides, photogrammetry can not only get the model but also extract the texture(Marc. 2015). It might bring about a huge advantage in architectural design. On the other hand, a convenient tool for customizing the path of the drone can also facilitate the research about how to plan better drone path per se; it is crucial for improving the quality of the model by photogrammetry. There are some cases about developing an algorithm to plan drone path, such as the research that plan the path for the plane, dealing with the more sophisticated task than hovering the helicopter(Joao. 2014). Besides, artists also begin viewing drone as a new media for their work, such as drawing pattern or image in the sky(Russell Klimas. 2019). On the other hand, photogrammetry is also suitable for documenting the current issue because it provides a 3D model that can be surveyed from various perspective(Stuart. 2016).

Those cases shows the significant potential of planning the drone path. Hence, it is worth developing a more convenient and versatile process for it. Since the user is able to integrate GPX and OpenStreetMap information more easily by using this tool, the user can utilize GPX application to measure the rough outline of the subject, planning and optimizing drone path in the parametric design environment. In this demonstration, we propose the approach to avoid missing certain detail of the building when taking photos in the sky. The path of the drone is optimized by focusing more on these part on the outline with relative poor visibility. For example, the concave part of a curve might cause occlusion, causing the defect. The majority of planning path application software doesn't deal with it. This tool makes the test and planning of drone path more
Figure 11

Converting GPX data into points in Rhino.

Figure 12 Using this tool for drone path planning. 
convenient. The following is the process from measuring rough outline to export waypoints onto drone application(litchi).

First of all, the user can acquire GPX data by using GPX applications on a mobile device. Then, the points of the rough outline can be derived from the GPX data with this tool(Figure 11).

The next step is drawing an offset outline from the original outline as a preliminary path. The offset outline can keep the particular distance from the building, making the scale of the features on building even. Then, the visibility of each part of the outline for a drone can be analyzed in Grasshopper. These parts with poor visibility might cause the defect; therefore, it is better to make the drone camera pay more attention to these parts. After rearranging the directions of positions, the subsequent step is converting positions and directions in Grasshopper into the waypoint format. The parameters that the drone path needs are the latitude, longitude, altitude, heading, and gimbal pitch. The latitude, longitude, and altitude are derived from the points in grasshopper; besides, the heading and gimbal pitch are derived from the vector of direction. Then, we use grasshopper for string handling according to the format that fits litchi hub("flylitchi". 2019). After generating CSV file for litchi, the user can import the file into Mission Hub- Litchi, upload onto the Litchi app on a mobile device. Then, the drone can be operated for photogrammetry(Figure 12).

\section{CONCLUSION}

In this research, the tool for facilitating the design process is developed by considering the way that architects employ tools. That's the reason why this tool is so compact instead of scattered. From then on, architects can utilize OSMKIT for integrating various information. Besides, architects are able to acquire the most current model without suffering from seeking models by hook or by crook. They can just employ their own drone with photogrammetry technology. Moreover, this tool further democratizes the process of contributing to OpenStreetMap, encouraging the relationship between user and data. The example of data visualization and planning drone path illustrates how to integrate several media, showing how the workflow can be. Additionally, the cause makes this tool handy is that it contains two main functions simultaneously; the first one is acquiring open Geographic information and the second is bilateral Conversion between points in Grasshopper and Geographic coordinate. To sum up, this tool links up the Grasshopper to the earth, providing a more intuitive solution to integrate various information.

\section{FUTURE WORK}

Although these functions and usages in this research are so practical for architecture, the potential for entertainment or art is worth thinking through. The future work would be the improvement of efficiency and multifunctional. The compatibility of the SHP format will be included in the next update. Furthermore, the next project that we take into account is the performance of drone swarm in grasshopper.

\section{ACKNOWLEDGMENT}

This research was supported by the Ministry of Science and Technology under Grant Number 1082634-F-009-013-, and was managed by the Pervasive Artificial Intelligence Research (PAIR) Labs, Taiwan.

\section{REFERENCES}

Florina, Dutt and Das, Subhajit 2016 'Geospatial Tool Evaluating Job Location Mismatch, Based on Available Workforce and Transit Options - Evaluating property location in a city using large-scale datasets', Proceedings of ASCAAD 2016, pp. 557-566

Gatziolis, Demetrios, Lienard, Jean, Vogs, Andre and Stringul, Nikolay 2015, '3D Tree Dimensionality Assessment Using Photogrammetry and Small Unmanned Aerial Vehicles', PloS one, 10, pp. 1-14

GREENWOOD, FAINE 2015, How to make maps with drones, drones.newamerica.org

Habermann, Marc (eds) 2015, Drone Path Planning, Saarland University

Hawkins, Stuart 2016 'Using a drone and photogrammetry software to create orthomosaic images and 3D models of aircraft accident sites', ISASI 2016 
Kunze, Antje, Marz, Michael and Wyka, Edyta 2018'Smart Communities', Proceedings of eCAADe 2018

LEE, Jung Hoon and KAGA, Atsuko 2013 'VISUALANALYSIS OFTHE RELATION BETWEEN CONCENTRATED DISTRICTS OF KNOWLEDGE-BASED INDUSTRIES AND THIRD PLACES IN OSAKACITY', Proceedings of CAADRIA 2013

Nagy, Danil, Stoddart, Jim, Villaggi, Lorenzo, Burger, Shane and Benjamin, David 2018 'Digital Dérive: Reconstructing Urban Environments based on Human Experience', Proceedings of ACADIA 2018

Panagoulia, Eleanna 2017"The role of Open Data in identifying and evaluating the Livability of Urban Space', Proceedings of eCAADe 2017

RYU, JUNGRIM and CHO, SEUNGYEON 2015 'A DEVELOPMENT DIRECTION OF A NEW ARCHIURBAN INTEGRATION MODEL FOR UTILIZING SPATIAL INFORMATION', Proceedings of caadria 2015

Schulz, Daniel, Reiter, Felix, Metche, Alexander and Werner, Liss 2018 'Data Flow: a GIS based interactive planning tool for educational facilities', Proceedings of eCAADe 2018

Valente, Joao 2014, Aerial Coverage Path Planning applied to Mapping, Ph.D. Thesis, Polytechnic University of Madrid

ZHONG, CHEN, ARISONA, STEFAN MÜLLER and SCHMITT, GERHARD 2014 'A VISUAL ANALYTICS FRAMEWORK FOR LARGE TRANSPORTATION DATASETS', Proceedings of CAADRIA 2014

[1] https://flylitchi.com/hub

[2] https://www.dailymail.co.uk/sciencetech/article-66

94741/Vincent-van-DRONE.html 